\title{
State Feedback Control in System of Rikitake Double Disk Dynamos
}

\author{
Jian Huang ${ }^{1, \mathrm{a}}$ \\ ${ }^{1}$ Department of Electronics and Information, Xijing University, Xi' an \\ a565200245@qq.com,
}

\begin{abstract}
As a nonlinear dynamical system of double-disc generator, double generators will produce chaos and limit cycle phenomenon under certain conditions and a range of external inputs.Using the state feedback control method control motion chaotic system. The feedback gain is determined by the pole placement method, and therefore it has a good dynamic response characteristics. Simulation results verify the validity of theoretical analysis and the effectiveness of the control method, as well as provide a theoretical reference for the study of excellent method for controlling.
\end{abstract}

Keywords-Two-Disk Dynamo ; Chaos Control ; State Variable Feedback

\section{INTRODUCTION}

In the 1960s, Lorenz discovered the "Butterfly Effect" in weather forecasting models. Since then, many researchers came into the relevant studies of chaos in nonlinear field [1].With the exploration of chaos research, the research value and application value was gradually realized in the chaos, especially in the motor system [2-5].According to the chaotic characteristics, many methods of research and analysis are proposed, providing theoretical basis and scientific analysis method for human understanding of nature [2].

Polarity reversal of geomagnetic secular variation in the geomagnetic theory is one of the problems, which need to be solved and is the most difficult to explain. In this regard, geomagnetic researchers made many model descriptions. Disk dynamo as the earliest origins of the geomagnetic and reversal simulation played a very important role. In 1958, the model of dynamo first proposed by Rikitake disk and the model with abundant chaos phenomenon was validated [6]. In recent years, double disk generator model has been applied to the project. In document [7], chaotic motion of the system is analyzed using a proportional-derivative control method, but it is difficult to achieve in the actual system. So far, he permanent magnet synchronous motor control methods have been reported and studied for many times, but the specific disk dynamo effective control method for chaotic systems are rarely reported. Therefore, between many control methods, using an effective control method of the system to avoid chaos becomes very important and has the practical significance [8].

In this paper, for the double-disc generator of chaotic systems, the basic dynamics of the chaotic system are analyzed using phase diagram, bifurcation diagram. Its abundant chaotic dynamics is verified. Considering the control method can effectively be applied to the project, we use a linear state feedback control method for
Rikitake chaotic movement of the generator control, and validate control strategies through MATLAB simulation.

\section{MATHEMATICAL MODEL AND CHAOS OF DOUBLE GENERATORS}

Rikitake disk first proposed the model of dynamo in 1958. Double generators are coupled by two discs and two coils together. Dimensionless equations of the model [6] are:

$$
\left\{\begin{array}{l}
\frac{d x_{1}}{d t}=-\mu x_{1}+x_{2} x_{3} \\
\frac{d x_{2}}{d t}=-\mu x_{2}+x_{1}\left[x_{3}-\mu\left(\sigma^{2}-\sigma^{-2}\right)\right] \\
\frac{d x_{3}}{d t}=1-x_{1} x_{2}
\end{array}\right.
$$

Among that, ${ }_{1}, x_{2}$ are dimensionless current, ${ }^{x_{3}}$ is the angular velocity of two disk, and $\mu$ and $\sigma$ are positive adjustable parameters. Let parameter be $\mu=1, \sigma=2$. The attractor of the system is shown in Fig. 1:

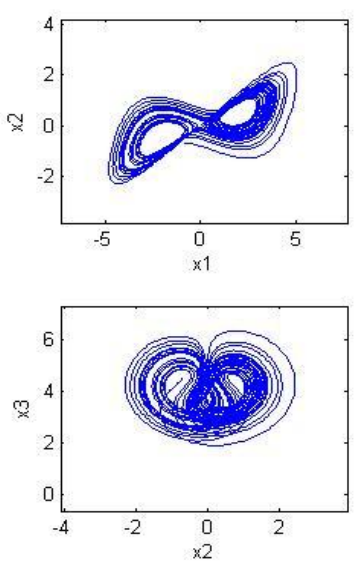

Fig. 1. Phase Diagram of the System

\section{THE INFLUENCE OF PARAMETERS ON THE SYSTEM}

Parameters $\mu$ is influenced by environmental and working conditions and other factors have a great impact. With the change of parameters, the system exhibits 
nonlinear. When it meets $\mu \in[0.5,2.5]$, the system appears chaotic state as shown in Fig. 2.

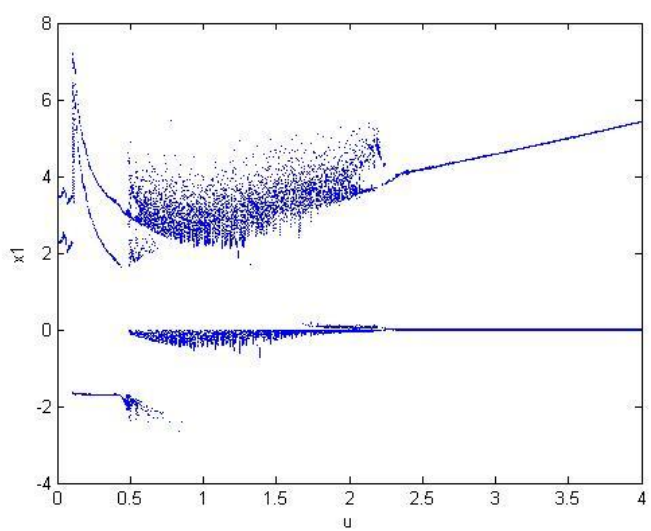

Fig 4. The Bifurcation Diagram of System

When the system is a chaotic motion, current and voltage are fluctuated, so it is very easy to lead the system to crash. Therefore, we need to find an appropriate and effective way to eliminate or suppress the chaotic behavior in the system.

\section{THE FEEDBACK CONTROL THEORY OF NONLINEAR SYSTEM STATE}

According to the literature in the [3] to the motor synchronous control of chaos, we can construct the response system. Consider the following nonlinear autonomous and out of control system.

$\frac{d X}{d t}=f(X, \alpha)$

Among them, $\mathrm{X}$ is state variable, and $\alpha$ is the parameters. The system (2) is approximated by:

$$
\frac{d X}{d t}=A(\alpha) X
$$

Among them, it has $A(\alpha)=\left.\frac{\partial f(X, \alpha)}{\partial X}\right|_{X=E_{0}}$.Control the state feedback of system (3). Therefore, the controlled system is:

$$
\frac{d X}{d t}=A(\alpha) X+B u
$$

Where in, $\mathrm{B}$ is a diagonal matrix, and $u$ is the control vector.

$$
u=-K\left(X-E_{0}\right)
$$

Where, $\mathrm{K}$ is a feedback gain matrix. [17].

In this paper, the method of pole assignment control theory to determine the solution of the $\mathrm{K}$ matrix is used, so that the system reaches steady state.
According to the theory, considering the system (1) with state feedback control, we get the controlled system of system (1).

$$
\left\{\begin{array}{l}
\frac{d x_{1}}{d t}=-\mu x_{1}+x_{2} x_{3}-k_{1}\left(x_{1}-x_{10}\right) \\
\frac{d x_{2}}{d t}=-\mu x_{2}+x_{1}\left[x_{3}-\mu\left(\sigma^{2}-\sigma^{-2}\right)\right]-k_{2}\left(x_{2}-x_{20}\right) \\
\frac{d x_{3}}{d t}=1-x_{1} x_{2}-k_{3}\left(x_{3}-x_{30}\right)
\end{array}\right.
$$

According to equation (4), B can be simplified. Let $B=\operatorname{diag}(1,1,1)$

In equilibrium balance in system (1), the matrix Jacbian of system (6) is:

$$
J=\left(\begin{array}{ccc}
-\mu-k_{1} & x_{30} & x_{20} \\
\mu \sigma^{-2} & -\mu-k_{2} & x_{10} \\
-x_{20} & -x_{10} & -k_{3}
\end{array}\right)
$$

Characteristic equation of the controlled system is:

$$
f(\lambda)=\lambda^{3}+A_{2} \lambda^{2}+A_{1} \lambda+A_{0}
$$

Among them, $A_{1}, A_{2}, A_{0}$ are associated with $k_{1}, k_{2}, k_{3}$.

Take the expected pole and the characteristic polynomial composed of the pole as:

$$
f(s)=s^{3}+12 s^{2}+21 s+10
$$

The characteristic polynomial (9) of pole is the same as the controlled system characteristic polynomial (8).It can be obtained $k_{1}=9, k_{2}=0, k_{3}=1$.When the system is running to 20 s, adding control, the simulation results is in fig .3.From fig .3 and 6,it can be found the controller is worked after 20 s, the system quickly reaches a steady state with good stability. 

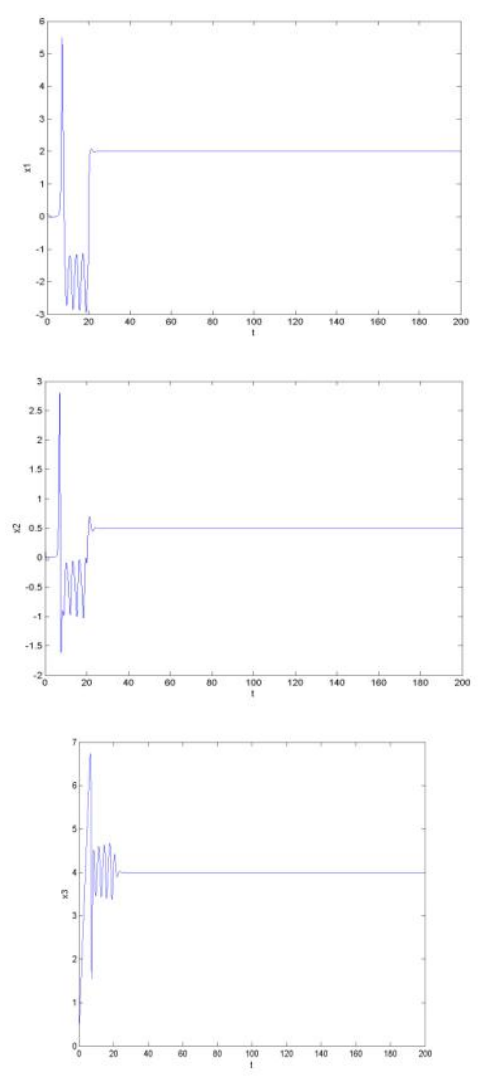
(a) state $x_{1}$ variables
(b) state ${ }^{x_{2}}$ variables

state $^{x_{3}}$ variables

Fig 3. The State of Motion of the Controlled System

\section{CONCLUSION}

This paper studies the Rikitake disk dynamo model. Studies have shown that the model has chaotic motion of the system in a certain range of parameters. In addition, the basic characteristics of chaos are also analyzed. For the chaotic characteristics of the generator, the state feedback control is applied. The whole system simulation studies show that the system can quickly reach a steady state under the action of the controller. Meanwhile, the theoretical analysis and numerical simulation results are consistent. The design of the control system does not require any additional information other than the system state variables of the controlled system. Research results show that this method is effective, and therefore the project has important reference value.

\section{REFERENCES}

[1] Lorenz E N. Deterministic nonperiodic flow [J].J Atmos.Science, 1963, 20:130-141.

[2] LIU Ting-ting, TAN Yu, WU Gang, et al. Simulating of high speed PMSM control system based on SVPWM [J]. Power System Protection and Control, 2009, 37(12): 11-14.

[3] WANG Lingzhi. Simulation of improved direct torque control system for permanent magnet synchronous motor [J]. Power System Protection and Control, 2009, 37(19): 65-69.

[4] WU Zhongqiang, AO Dun. The tracking chaos control of a linear continuous system-T-S model method [J]. Electric Machines and Control, 2007, 11(2): 201-206.

[5] CHEN Min, LI Ze-jun, LI Ang. Forecast research of urban electricity consumption based on Chaos theory [J]. Power System Protection and Control, 2009, 37(16): 41-45.

[6] Keisuke I. Chaos in the Rikitake two disk dynamo system [J]. Earth and Planetary Science Letters, 1980, 51(2): 451 - 456

[7] CHANG Yingchun, LIU Xiaojun, LI Xianfeng. chaos and chaos control of the rikitake Two-Disk Dynamo[J].Journal liao ning Normal University (Natural science Edition)2006. 29 (4) : 422-426

[8] WANG Fa-qiang, LIU Chong-xin. Studies on Liu chaotic system and its experimental confirmation [J]. Acta Physica Sinica, 2006, 